\title{
Security-Enhanced OSGi Service Environments
}

\author{
Gail-Joon Ahn, Senior Member, IEEE, Hongxin Hu, and Jing Jin
}

\begin{abstract}
Today's home and local-area network environments consist of various types of personal equipments, network devices, and corresponding services. Since such prevalent home network environments frequently deal with private and sensitive information, it is crucial to legitimately provide access control for protecting such emerging environments. As a result, the open services gateway initiative (OSGi) attempted to address this critical issue. However, the current OSGi authorization mechanism is not rigorous enough to fulfill security requirements involved in dynamic OSGi environments. In this paper, we provide a systematic way to adopt a role-based access control (RBAC) approach in OSGi environments. We demonstrate how our authorization framework can achieve important RBAC features and enhance existing primitive access control modules in OSGi service environments. Also, we describe a proof-of-concept prototype of the proposed framework to discuss the feasibility of our approach using an open source implementation of OSGi framework known as Knopflerfish.
\end{abstract}

Index Terms-Authorization, OSGi service, role-based management, security.

\section{INTRODUCTION}

A $\mathrm{S}$ we find ourselves accustomed to the pervasiveness and prevalent availability of network services, the proliferation of inexpensive digital equipments and the Internet have expedited such a rapid growth of availability to a scale scarcely imagined a few years ago. However, with the continuous connectivity to the Internet, home devices are exposed to various attacks and threats. The traditional security technologies cannot satisfy security requirements derived from a digital home environment because of the diversity of wired and wireless network, middleware modules, and restricted system resources of home appliances. Also, the users in a digital home environment mostly lack knowledge on security and network management practices [10], [13]. Just as technological innovation has enabled tremendous availability for home network environments, it is crucial to legitimately provide access control for protecting devices and digital resources in such environments. In the networked systems, access control can prescribe not only who or what process has access to a specific system resource, but also the type of access that should be granted.

Manuscript received August 24, 2008; revised January 7, 2009. First published May 15, 2009; Current version published August 19, 2009. This work was supported in part by the funds provided by the National Science Foundation under Grant NSF-IIS-0242393 and the Department of Energy Early Career Principal Investigator Award under Grant DE-FG02-03ER25565. This paper was recommended by Associate Editor N. Wu.

G.-J. Ahn and H. Hu are with the Department of Computer Science and Engineering, Ira A. Fulton School of Engineering, Arizona State University, Tempe, AZ 85281-8809 USA (e-mail: gahn@asu.edu; hxhu@asu.edu).

J. Jin is with the Department of Software and Information Systems, University of North Carolina at Charlotte, Charlotte, NC 28223 USA (e-mail: jjin@uncc.edu).

Color versions of one or more of the figures in this paper are available online at http://ieeexplore.ieee.org.

Digital Object Identifier 10.1109/TSMCC.2009.2020437
The open services gateway initiative (OSGi) provides a framework to connect diverse devices in a local area network environment such as homes, offices, and automobiles. By defining the standard execution environment and service interface, OSGi promotes the dynamic discovery and collaboration of devices and services from different sources [3]. Moreover, this framework is designed to support connectivity to the outside users allowing remote control and administrative management [23]. In OSGi service platform, the User Admin service provides user authorization functionality. However, the User Admin service's authorization architecture in OSGi is not sufficient enough to support the highly dynamic and open OSGi environments. Thus, an enhanced access control mechanism is needed for achieving interoperability, extensibility, and ease of administration and management. To overcome such issues, role-based access control (RBAC) can be considered to simplify authorization management by associating users with roles, and roles with permissions [7], [19], [24]. Because the roles within an organization typically have overlapping permissions, RBAC supports a hierarchical structure of roles to provide permission inheritance, where a senior role can inherit all permissions assigned to junior roles. As a fundamental aspect of RBAC, constraints can also allow us to lay out higher level access control policies [6], [11].

Recently, several research work [8], [9], [14] have addressed access control issues in OSGi environments using RBAC model. However, these research efforts did not consider the standardized efforts of OSGi authorization. Such generic approaches cannot accommodate all OSGi authorization requirements identified in the OSGi authorization standard [17], causing severe compatibility issues with existing OSGi applications. In addition, they failed to address important RBAC features such as constraints and role hierarchy construction related to OSGi environments. In this paper, we propose a systematic way to apply RBAC to OSGi environments. In our proposed approach, the OSGi authorization mechanism is configured and mapped to RBAC, demonstrating that OSGi authorization requirements can be fully fulfilled by RBAC. Furthermore, we incorporate important RBAC features, which were not addressed by current OSGi authorization mechanism, to enhance existing access control modules in OSGi service environments. Also, a prototype system is implemented to demonstrate the feasibility and effectiveness of our proposed solution.

The rest of this paper is organized as follows. In Section II we briefly describe OSGi service platform, OSGi authorization mechanism, RBAC, and related work. Section III gives an overview of our authorization framework. We also formalize OSGi authorization mechanism and show how OSGi authorization can be accommodated in RBAC. In addition, we discuss the reconstruction of OSGi authorization from RBAC to verify the completeness of our approach. The implementation of our 
prototype is described in Section IV. Section V concludes the paper and discusses the future work.

\section{BACKGROUND AND RELATED WORK}

\section{A. OSGi Service Platform}

OSGi specifies an open, common architecture for service providers, developers, software venders, gateway operators, and equipment vendors to develop, deploy, and manage services in a cooperative fashion. In addition, OSGi service platform is an extensible integration platform used to remotely and dynamically deploy, provide, maintain, and manage applications and services with multiple devices in networked environments [3]. We first overview the architecture of OSGi service platform. The architecture of OSGi service platform is composed of a set of bundles based on the OSGi framework that provides the basic functionalities to perform OSGi functions through downloading and executing bundles. Each bundle is a software component that contains algorithms and protocols for controlling a device. When OSGi service gateway requests a bundle, it can be read from local disk or retrieved from the repository and then executed by the service gateway. In other words, OSGi service platform allows bundles to be configured dynamically. This feature enables a home network environment to download and utilize the latest and most optimal bundles, and provides customization of gateway functions for each user taking into account individual profiles.

In OSGi service platform, the User Admin service provides the authorization functionality [17]. All bundles should use the User Admin service to find out if the users attempting to access are authorized or not. In the User Admin service authorization architecture, three components are defined as follows.

1) User-A user is a human being who can be identified by credentials such as a password and other identity attributes.

2) User Group - A user group is an aggregation of users based on common properties. For example, all family members belong to a user group named Residents.

3) Action Group-Every action that can be performed by a bundle is associated with an action group. For example, if a bundle could be used to control the alarm system, there should be an action group named AlarmSystemControl.

In OSGi authorization, the authorization decision is made based on the following two strategies.

1) ANY Strategy: A user could be allowed to carry out an action if he/she belongs to Any member of the action group. For example, the AlarmSystemControl action group contains two user groups Administrators and Residents. Elmer, Pepe, and Bugs belong to Administrators user group, and Elmer, Pepe, and Daffy belong to Residents user group as follows:

$$
\begin{aligned}
\text { Administrators } & =\{\text { Elmer, Pepe, Foghorn }\} \\
\text { Residents } & =\{\text { Elmer, Pepe, Daffy }\}
\end{aligned}
$$

AlarmSystemControl $=\{$ Administrators, Residents $\}$.
TABLE I

USER GROUPS WITH BASIC USER MEMBERS

\begin{tabular}{|c|c|c|c|c|c|c|}
\hline & Elmer & Fudd & Marvin & Pepe & Daffy & Foghorn \\
\hline \hline Residents & Basic & - & - & Basic & Basic & - \\
\hline Buddies & - & - & - & - & Basic & Basic \\
\hline Children & - & - & Basic & Basic & - & - \\
\hline Adults & Basic & Basic & - & - & - & Basic \\
\hline Administrators & Basic & - & - & Basic & - & Basic \\
\hline
\end{tabular}

TABLE II

ACTION GROUPS WITH BASIC AND REQUIRED USER GROUP MEMBERS

\begin{tabular}{|l|l|l|l|l|l|}
\hline & Residents & Buddies & Children & Adults & Admins \\
\hline \hline AlarmSystemControl & Basic & - & - & - & Required \\
\hline InternetAccess & Basic & - & Basic & Basic & - \\
\hline TemperatureControl & Required & - & - & Required & - \\
\hline WebCamAccess & Basic & Basic & - & Required & Required \\
\hline PhotoAlbumView & Basic & Basic & - & - & - \\
\hline
\end{tabular}

This ANY strategy allows any of four members, Elmer, Pepe, Foghorn, and Daffy, to activate the alarm system since all users are a member of user groups and these user groups belong to one action group.

2) ALL Strategy: A user is allowed to carry out an action if he/she belongs to All members of the action group. In the aforementioned AlarmSystemControl example, only Elmer and Pepe would be authorized to activate the alarm system, since Daffy and Bugs are not members of both the Administrators and Residents user groups.

The implementation of User Admin service in OSGi service platform supports a combination of both strategies by introducing two member sets, namely the basic member set for the ANY strategy and the required member set for ALL strategy. Basic membership defines a set of members that can get access, and required membership reduces this set by requiring the user to be a required member of each action group.

To accommodate this, OSGi allows to assign a user to a user group, and then, the user group is assigned to a specific action group. Tables I and II show an authorization example to demonstrate the assignment relationships, respectively.

The access decision is made based on the basic and required assignment relationships. For example, in Table II, the action group WebCamAccess has two basic members, Residents and Buddies, and two required members, Adults and Administrators. Thus, all users belonging to at least one of the basic members, Residents and Buddies, and all required members, Adults and Administrators, are able to carry out the webcam access action. From Table I, we can see two users Elmer and Foghorn can meet this authorization requirement.

\section{B. Overview of RBAC Model}

RBAC is an alternative policy to traditional mandatory access control (MAC) and discretionary access control (DAC) [22]. As MAC is used in the classical defense arena, the policy of access is based on the classification of objects such as security clearance [21]. The main idea of DAC is that the owner of an object has discretionary authority over the one who can access that object [12], [20]. But RBAC policy is based on the role of the subjects and can specify security policy in a way that maps to an organization's structure. 
A general family of RBAC models called RBAC96 was defined by Sandhu et al. [19]. Intuitively, a user is a human being or an autonomous agent, a role is a job function or job title within the organization with some associated semantics regarding the authority and responsibility conferred on the user assigned to the role, and a permission is an approval of a particular mode of access to one or more objects in the system or some privilege to carry out specified actions. Roles are organized in a partial order $\geq$, so that if $x \geq y$, then role $x$ inherits the permissions of role $y$. Members of $x$ are also implicitly members of $y$.

\section{Related Work}

Several related work studies the access control mechanism for home network environments where the OSGi service platform is operated. Cho et al. [8], [9] proposed an authorization policy management framework based on RBAC for OSGi service platform. Through the comparison of several access control models, especially DAC and RBAC, they claimed that RBAC model is flexible and more proper than DAC model for home network environments operated by the OSGi service platform. However, they did not consider the established authorization mechanism in current OSGi standard and omitted how RBAC can be effectively utilized to meet the OSGi authorization requirements. Also, their approach disregarded important RBAC features, such as role hierarchy and constraints. Our approach formalizes current OSGi authorization mechanism, and presents a systematic and comprehensive way to map OSGi authorization mechanism to RBAC configuration so that OSGi authorization requirements can be fully satisfied and enhanced by RBAC. Lim et al. [14] presented a mechanism to bundle authentication and authorization services using XML for the OSGi service platform. Their proposed approach uses extensible access control markup language (XACML) to specify RBAC polices for the authorization of service bundles. However, they also ignored existing OSGi authorization mechanism and mainly attempted to deploy the notion of roles in the OSGi platform directly without considering essential RBAC features. To the best of our knowledge, our approach illustrated in this paper is the first RBAC authorization solution that is compatible to the existing OSGi authorization standard, allowing the OSGi application developers and system administrators to better understand and manage security policies.

\section{OUR APPROACH}

We witness that the current OSGi authorization mechanism is not intuitive for authorization administration. Especially, current OSGi mechanism is not suitable for satisfying all security requirements for defining fine-grained access control policies in a highly dynamic and open OSGi environment. Our objective is to provide an efficient and effective authorization mechanism for such network environments. To achieve this, we propose a rolebased authorization framework for OSGi service environments. Our authorization framework consists of following processes.

1) Mapping OSGi authorization mechanism to RBAC: RBAC is a powerful mechanism for reducing the management complexity, administration cost, and potential configura- tion error within the organization. To achieve such features, it is inevitable to identify and derive RBAC components from OSGi authorization requirements. This identification phase allows us to discover roles, role hierarchy, assignment relations, and constraints. Those identified components from OSGi authorization requirements are used to construct a RBAC-based authorization environment that fully simulates the OSGi authorization environment, and provides more fine-grained and robust authorization services.

2) Reconstructing OSGi authorization from RBAC: Through mapping OSGi authorization mechanism to RBAC, the complexity of OSGi authorization management is reduced and OSGi authorization can be enhanced by adopting important RBAC features. In addition, we need to validate whether changes in the RBAC-based environment can also be reflected in OSGi authorization environment. In other words, our constructed RBAC environment should be converted back to the OSGi authorization environment, assuring those changes remain intact.

We attempt to formally define components in the current OSGi authorization mechanism, and these formal definitions are used through the rest of this paper. There are three sets of entities: users $(U)$, user groups (UG), and action groups (AG). The basic user-to-user group assignment (BUA) is a manyto-many relation between $U$ and UG. There are two kinds of many-to-many relation between UG and AG. One is basic action group-to-user group assignment (BAA) that reflects the basic memberships for each action. Another is required action group-to-user group assignment (RAA) that reflects the required memberships for each action. The following definitions formally summarize each component.

1) $U$ is a set of users, $U=\left\{u_{1}, \ldots, u_{n}\right\}$.

2) $\mathrm{UG}$ is a set of user groups, $\mathrm{UG}=\left\{\operatorname{ug}_{1}, \ldots, \mathrm{ug}_{n}\right\}$.

3) $\mathrm{AG}=\mathrm{AG}_{\mathrm{Basic}} \cup \mathrm{AG}_{\text {Required }}$ is a set of basic and required action groups, $\mathrm{AG}=\left\{\mathrm{ag}_{1}, \ldots, \mathrm{ag}_{n}\right\}$.

4) BUA $\subseteq U \times$ UG, is a many-to-many basic user-to-user group assignment relation.

5) $\mathrm{BAA} \subseteq \mathrm{AG}_{\mathrm{Basic}} \times \mathrm{UG}$, is a many-to-many basic action group-to-user group assignment relation.

6) $\mathrm{RAA} \subseteq \mathrm{AG}_{\text {Required }} \times \mathrm{UG}$, is a many-to-many required action group-to-user group assignment relation.

7) users: $U G \rightarrow 2^{U}$ is a function mapping each user group $\operatorname{ug}_{i}$ to a set of users users $\left(\operatorname{ug}_{i}\right)=\left\{u \in U \mid\left(u, \operatorname{ug}_{i}\right) \in\right.$ BUA\}.

8) user_groups: $U \rightarrow 2^{\mathrm{UG}}$ is a function mapping each user $u_{i}$ to a set of user groups user_groups $\left(u_{i}\right)=\{\operatorname{ug} \in$ $\left.\mathrm{UG} \mid\left(u_{i}, \mathrm{ug}\right) \in \mathrm{BUA}\right\}$.

9) ba_user_groups: $A_{\text {Basic }} \rightarrow 2^{U G}$ is a function mapping each basic action group $\mathrm{ag}_{i}$ to a set of user groups, ba_user_groups $\left(\mathrm{ag}_{i}\right)=\left\{u g \in \mathrm{UG} \mid\left(\mathrm{ag}_{i}, \mathrm{ug}\right) \in \mathrm{BAA}\right\}$.

10) re_user_groups: $\mathrm{AG}_{\mathrm{Required}} \rightarrow 2^{\mathrm{UG}}$ is a function mapping each required action group $\mathrm{ag}_{i}$ to a set of user groups, re_user_groups $\left(\mathrm{ag}_{i}\right)=\left\{\mathrm{ug} \in \mathrm{UG} \mid\left(\mathrm{ag}_{i}, \mathrm{ug}\right) \in \mathrm{RAA}\right\}$.

11) ba_action_groups: $U G \rightarrow 2^{A G_{B} \text { asic }}$ is a function mapping each user group ug $_{i}$ to a set of basic action groups, 
ba_action_groups $\left.\left(\operatorname{ug}_{i}\right)\right]=\left\{\mathrm{ag} \in \mathrm{AG}_{\mathrm{Basic}} \mid\left(\mathrm{ag}, \mathrm{ug}_{i}\right) \in\right.$ BAA .

12) re_action_groups: $U G \rightarrow 2^{A G_{R e q u i r e d}}$ is a function mapping each required user group $\mathrm{ug}_{i}$ to a set of required action groups, re_action_groups $\left(\operatorname{ug}_{i}\right)=\{\mathrm{ag} \in$ $\left.\mathrm{AG}_{\text {Required }} \mid\left(\mathrm{ag}, \mathrm{ug}_{i}\right) \in \mathrm{RAA}\right\}$.

\section{A. Construction of OSGi-Compliant RBAC}

In this section, we demonstrate how RBAC components and features can be utilized to support components of OSGi authorization. We describe how we can identify basic RBAC component, role hierarchy, and constraints to enforce OSGi authorization requirements.

1) Basic RBAC Components Construction: The essence of RBAC is the notion of roles [24] to abstract users and permissions. Permissions are grouped through roles, and users obtain permissions by being assigned to roles. Users, roles, permissions, and the corresponding assignment relations are core components in RBAC.

We noted that the user set $(U)$ and the action group set (AG) can essentially be treated as users and permissions in the RBAC model, respectively. In addition, the user group set (UG) in OSGi can be represented as roles such that actions are abstracted through user groups, and these actions can be exercised by a member of the specific user group. However, normally OSGi uses two types of assignments, namely, the basic assignment (BAA) and the required assignment (RAA) that can allow us to achieve the abstraction of actions through user groups. However, RBAC cannot accommodate this characteristic directly, so it leads us to propose several properties to bridge this gap in the course of OSGi-compliant RBAC construction.

First, we construct the role $(R)$ by introducing a concept of Private Membership. In particular, we treat the user groups in OSGi as the private members of each role in role construction, which can be further characterized as basic members and required members depending on the property of the OSGi BAA relation and RAA relation. The detailed role construction process is explained through an OSGi authorization example, as shown in Fig. 1. In this example, Table (a) reflects the assignment relation between $U$ and $\mathrm{UG}$, and Table (b) shows the assignment relation between AG and UG. In Table (b), there are two types of assignment relations, Basic and Required, to reflect BAA and RAA relations, respectively. For a particular action group, we identified that there exist three possible combinations on Basic and Required assignments given as follows.

1) Case 1: Contains both basic assignments and required assignments, as shown in the row of $\mathrm{ag}_{1}, \quad$ ba_user_groups $\left(\mathrm{ag}_{1}\right)=\left\{\mathrm{ug}_{1}, \mathrm{ug}_{2}\right\} \quad$ and re_user_groups $\left(\mathrm{ag}_{1}\right)=\left\{\mathrm{ug}_{4}, \mathrm{ug}_{5}\right\}$.

2) Case 2 : Contains basic assignments only, as shown in the row of ag $\mathrm{ag}_{3}$, ba_user_groups $\left(\mathrm{ag}_{3}\right)=\left\{\mathrm{ug}_{1}, \mathrm{ug}_{2}, \mathrm{ug}_{3}\right\}$.

3) Case 3: Contains required assignments only as shown in the row of ag $\mathrm{g}_{2}$, re_user_groups $\left(a g_{2}\right)=\left\{\mathrm{ug}_{1}, \mathrm{ug}_{4}, \mathrm{ug}_{5}\right\}$.

As specified in the OSGi authorization rule, a user must be assigned to $A L L$ required user groups and $A N Y$ basic user groups before he/she can exercise a particular action. Also, while ac-

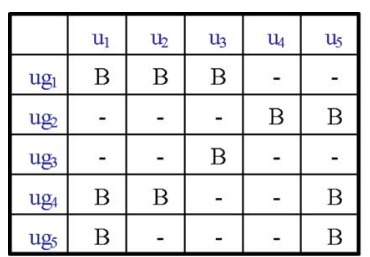

(a)

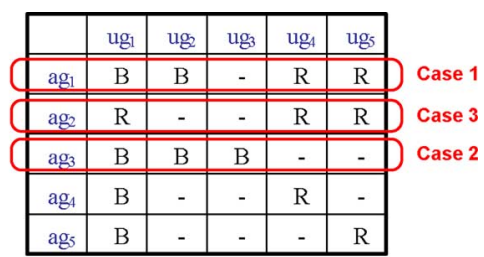

(b)
Fig. 1. OSGi authorization example. (a) USER-UG assignment. (b) AG-UG assignment.

commodating the identified basic and required assignment patterns, we define the following mapping rules on action group basis.

Rule 1: Introduce one role and one permission-to-role assignment (PA) for each OSGi basic action group-to-user group assignment in BAA. The role contains only one basic private member of the basic user group and ALL required members of the required user groups. The particular action group in BAA is constructed as one permission and the permission is assigned to the role.

Rule 2: In case of no basic assignment, introduce one role that contains ALL required members of the required user groups and one PA by assigning the permission to the role.

Rule 3: In terms of user-to-role assignment, a user can be assigned to a role when the corresponding user in OSGi authorization is assigned to all the private members of that role.

Now, we discuss how to use these three rules to construct the RBAC for each case that we have identified. First, we map all users and action groups in OSGi authorization to users and permissions directly as RBAC components. As identified in Case 1, the action group ag ${ }_{1}$ has both basic member and required member. Following Rule 1, we construct roles such as two roles $r_{1}$ and $r_{2}$ for the action group ag $\mathrm{g}_{1}$ in our example. Role $r_{1}$ contains a basic member ug $_{1}$, and two required members ug ug $_{4}$ and $\mathrm{ug}_{5}$. Role $r_{2}$ is constructed by a basic member $\mathrm{ug}_{2}$, and all required members $\mathrm{ug}_{4}$ and $\mathrm{ug}_{5}$. Formally, members $\left(r_{1}\right)=$ $\left\{\mathrm{ug}_{1}, \mathrm{ug}_{4}, \mathrm{ug}_{5}\right\}$ and members $\left(r_{2}\right)=\left\{\mathrm{ug}_{2}, \mathrm{ug}_{4}, \mathrm{ug}_{5}\right\}$. Using the same rule, the permission $p_{1}$ corresponding to the action group $\mathrm{ag}_{1}$ is assigned to roles $r_{1}$ and $r_{2}$. Following Rule 3, a user is assigned to a role only if the user has been assigned to all private members of this role. In the example, [see Fig. 1(a)], $u_{1}$ is assigned to $\mathrm{ug}_{1}, \mathrm{ug}_{4}$, and $\mathrm{ug}_{5}$. Since $\mathrm{ug}_{1}, \mathrm{ug}_{4}$, and $\mathrm{ug}_{5}$ are private members of $r_{1}, u_{1}$ should be assigned to $r_{1}$ in RBAC. For the same reason, $u_{5}$ is assigned to $r_{2}$. From Fig. 2, $u_{1}$ and $u_{5}$ can hold the same action group $\mathrm{ag}_{1}$, and $u_{1}$ and $u_{5}$ own the same permission $p_{1}$ via $r_{1}$ and $r_{5}$, respectively. Hence, the same authorization requirements are fulfilled through the RBAC configuration process.

In Case 2, the action group has only three basic members $u g_{1}, u_{2}$, and $u_{3}$. Using Rule 1 , three roles $r_{4}, r_{5}$, and $r_{6}$ are constructed by these three basic members of $\mathrm{ag}_{3}$, respectively, as shown in Fig. 2. Formally, members $\left(r_{4}\right)=$ $\left\{\operatorname{ug}_{1}\right\}$, members $\left(r_{5}\right)=\left\{\operatorname{ug}_{2}\right\}$, and members $\left(r_{6}\right)=\left\{\operatorname{ug}_{3}\right\}$. Corresponding assignment relations based on Rules 1 and 3 are shown as well. 


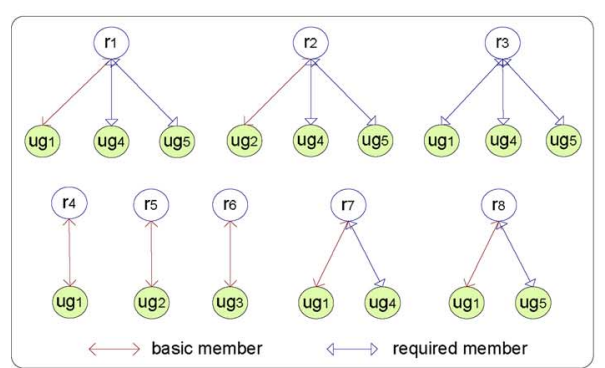

(a)

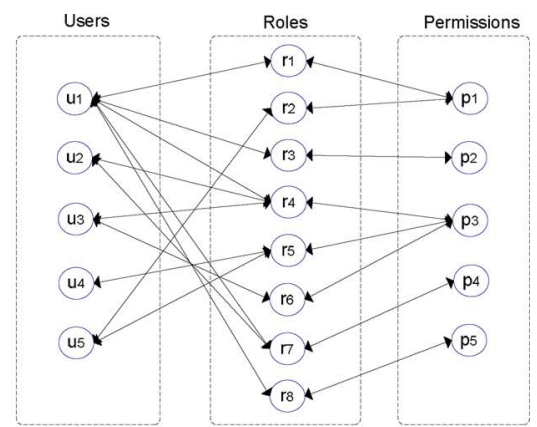

(b)

Fig. 2. Overall process of constructing RBAC from an OSGi authorization. (a) Role construction. (b) Mapping to RBAC.

TABLE III

FunCtions/PREDICATES USED IN THE PAPER

\begin{tabular}{|c|c|}
\hline Function/predicate & Description \\
\hline newRole $(\mathrm{bm}, \mathrm{RM})$ & Returns a new role constructed by a basic member $b m$ and/or all required members from $R M$. \\
\hline $\operatorname{addPA}(p, r)$ & Returns True if permission $p$ is assigned role $r$. \\
\hline $\operatorname{deletePA}(p, r)$ & Returns True if assignment form permission $p$ to role $r$ is deleted. \\
\hline $\operatorname{addUA}(u, r)$ & Returns True if user $u$ is assigned role $r$. \\
\hline isUA(u,r) & Returns True if user $u$ has been assigned role $r$. \\
\hline $\operatorname{deleteUA}(u, r)$ & Returns True if assignment form user $u$ to role $r$ is deleted. \\
\hline isSenior $\left(r_{i}, r_{j}\right)$ & Returns True if role $r_{i}$ is senior to role $r_{j}$. \\
\hline isJunior $\left(r_{i}, r_{j}\right)$ & Returns True if role $r_{i}$ is junior to role $r_{j}$. \\
\hline allSenior $(r)$ & Returns the set of all senior of role $r$ \\
\hline allJunior $(r)$ & Returns the set of all junior of role $r$ \\
\hline parents $(r)$ & Returns the set of all immediate senior of role $r$ \\
\hline children( $(r)$ & Returns the set of all immediate junior of role $r$ \\
\hline $\operatorname{addParent}\left(r_{i}, r_{j}\right)$ & Returns True if role $r_{i}$ is added to role hierarchy as an immediate senior of role $r_{j}$ \\
\hline $\operatorname{addChild}\left(r_{i}, r_{j}\right)$ & Returns True if role $r_{i}$ is added to role hierarchy as an immediate junior of role $r_{j}$ \\
\hline deleteParent $\left(r_{i}, r_{j}\right)$ & Returns True if role $r_{i}$ is deleted from role hierarchy as an immediate senior of role $r_{j}$ \\
\hline deleteChild $\left(r_{i}, r_{j}\right)$ & Returns True if role $r_{i}$ is deleted from role hierarchy as an immediate junior of role $r_{j}$ \\
\hline members $(r)$ & Returns the set of all private members of role $r$ \\
\hline ba_member $(r)$ & Returns the set of the basic private member of role $r$ \\
\hline re_members $(r)$ & Returns the set of all required private members of role $r$ \\
\hline addBUA(u, ug) & Returns True if user $u$ is assigned user group $u g$ as a basic member. \\
\hline deleteBUA(u,ug) & Returns True if basic assignment form user $u$ to user group $u g$ is deleted. \\
\hline $\operatorname{addBAA(ag,~ug)}$ & Returns True if action group $a g$ is assigned user group $u g$ as a basic member. \\
\hline $\operatorname{deleteBAA(ag,ug)}$ & Returns True if basic assignment form action group ag to user group $u g$ is deleted. \\
\hline $\operatorname{addRAA(ag,~ug)}$ & Returns True if action group $a g$ is assigned user group $u g$ as a required member. \\
\hline deleteRAA(ag, ug) & Returns True if required assignment form action group ag to user group $u g$ is deleted. \\
\hline
\end{tabular}

In Case 3, the action group has only required members. The second row of the OSGi authorization example [see Fig. 1 (b)] shows an action group $\mathrm{ag}_{2}$ that has three required members $\mathrm{ug}_{1}, \mathrm{ug}_{4}$, and $\mathrm{ug}_{5}$. Using Rule 2, one role $r_{3}$ is constructed that contains all three required members of $\mathrm{ag}_{2}$. Formally, members $\left(r_{3}\right)=\left\{\operatorname{ug}_{1}, \mathrm{ug}_{4}, \mathrm{ug}_{5}\right\}$. Fig. 2 depicts the role construction and assignment relationships.

In addition to the elements and functions in both our formal representation of OSGi authorization and the RBAC model, Table III lists additional functions and predicates which are used in the subsequent sections. To realize the basic RBAC construction from OSGi authorization, we develop a mapping algorithm, which maps OSGi authorization to basic RBAC specification. For the mapping algorithm in Fig. 3, let $n_{U}, n_{A G}$, and $n_{B A A}$ denote the number of users, action groups, and basic assignment relationships (between action groups and user groups), respectively. The computational complexity of this mapping algorithm is $O\left(n_{A G} n_{B A A} n_{U}\right)$.

2) Building Role Hierarchies: We construct role hierarchy using a NTree [18] structure to define an inheritance relationship, reducing the cost of administration. For example, all man- agers in the same organization may have a certain set of core "management privileges," even though they work in different departments. This commonality can be exploited through a role hierarchy that enables each department manager role to inherit a generic "management" role. Role hierarchy allows the policy designer to write generic access polices once and to simplify the complexity of access control policies.

In our mapping approach, roles are constructed based on $b a$ sic members and required members of action groups of OSGi authorization mechanism. Considering the private member sets of every role, we discover inclusion relations between them. For example, in Fig. 4(a), $r_{1}$ is constructed by a basic private member $\mathrm{ug}_{1}$, and two required private members $\mathrm{ug}_{4}$ and $\operatorname{ug}_{5}$. Formally, ba_member $\left(r_{1}\right)=\left\{\operatorname{ug}_{1}\right\}$ and re_members $\left(r_{1}\right)=$ $\left\{\operatorname{ug}_{4}, \operatorname{ug}_{5}\right\}$; while $r_{7}$ is constructed by a basic private member $\mathrm{ug}_{1}$ and a required private members $\mathrm{ug}_{4}$, i.e., ba_member $\left(r_{7}\right)=$ $\left\{\operatorname{ug}_{1}\right\}$ and re_members $\left(r_{7}\right)=\left\{\operatorname{ug}_{4}\right\}$. Hence, the following condition is true

$$
\begin{aligned}
& \left(\text { ba_member }\left(r_{7}\right)=\text { ba_member }\left(r_{1}\right)\right) \wedge \\
& \left(\text { re_members }\left(r_{7}\right) \subset \text { re_members }\left(r_{1}\right)\right) .
\end{aligned}
$$




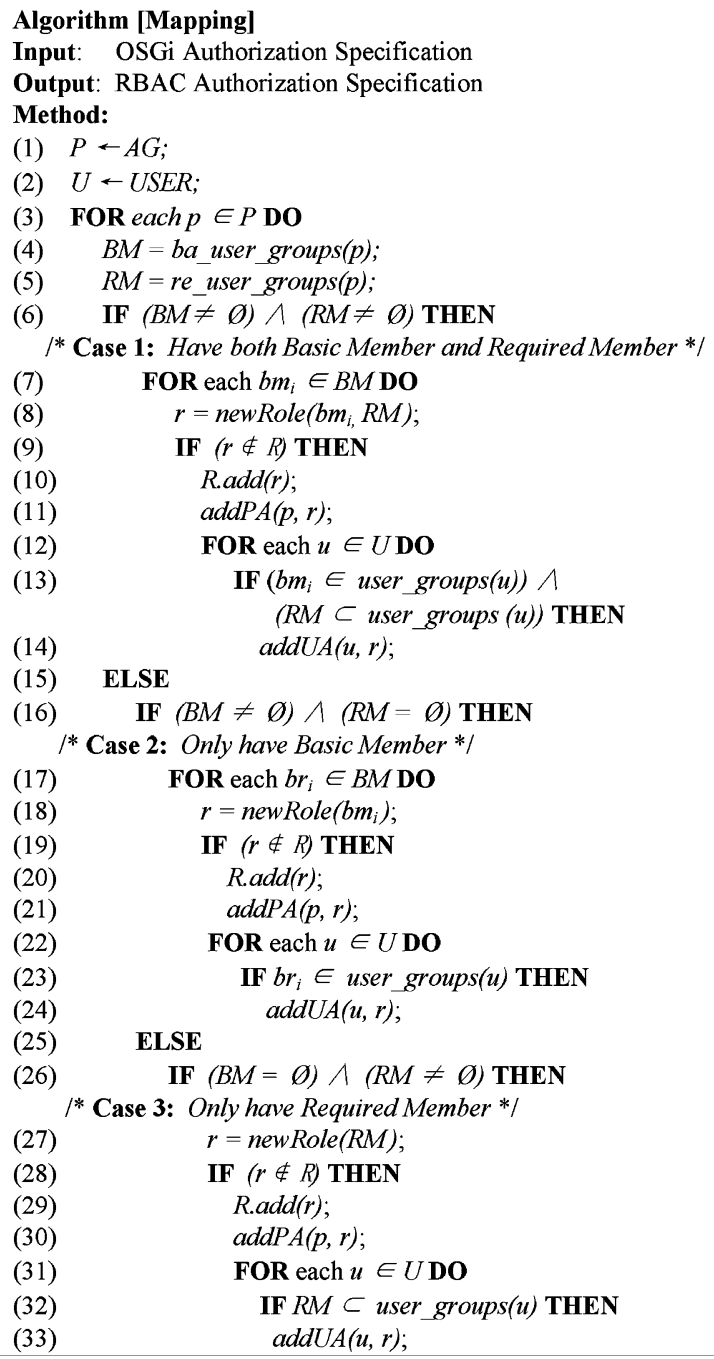

Fig. 3. Mapping algorithm from OSGi authorization to RBAC authorization.

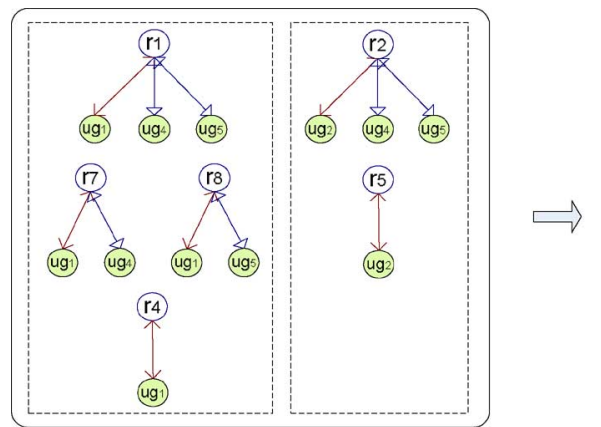

(a)

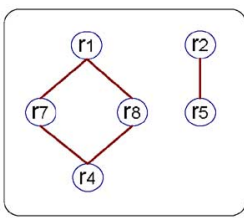

(b)
Fig. 4. Identifying role hierarchy relationships from role construction. (a) Role construction. (b) Role hierarchies.

Since the private members of $r_{1}$ include all private members of $r_{7}$, a user who is assigned to $r_{1}$ should be assigned to $r_{7}$ as well according to Rule 3 . Thus, a user assigned to $r_{1}$ should have all permissions of $r_{7}$. In other words, $r_{1}$ should possess all permissions of $r_{7}$, and $r_{1}$ is more powerful than $r_{7}$. Therefore, a role hierarchy relation can be build between $r_{1}$ and $r_{7}$. As a

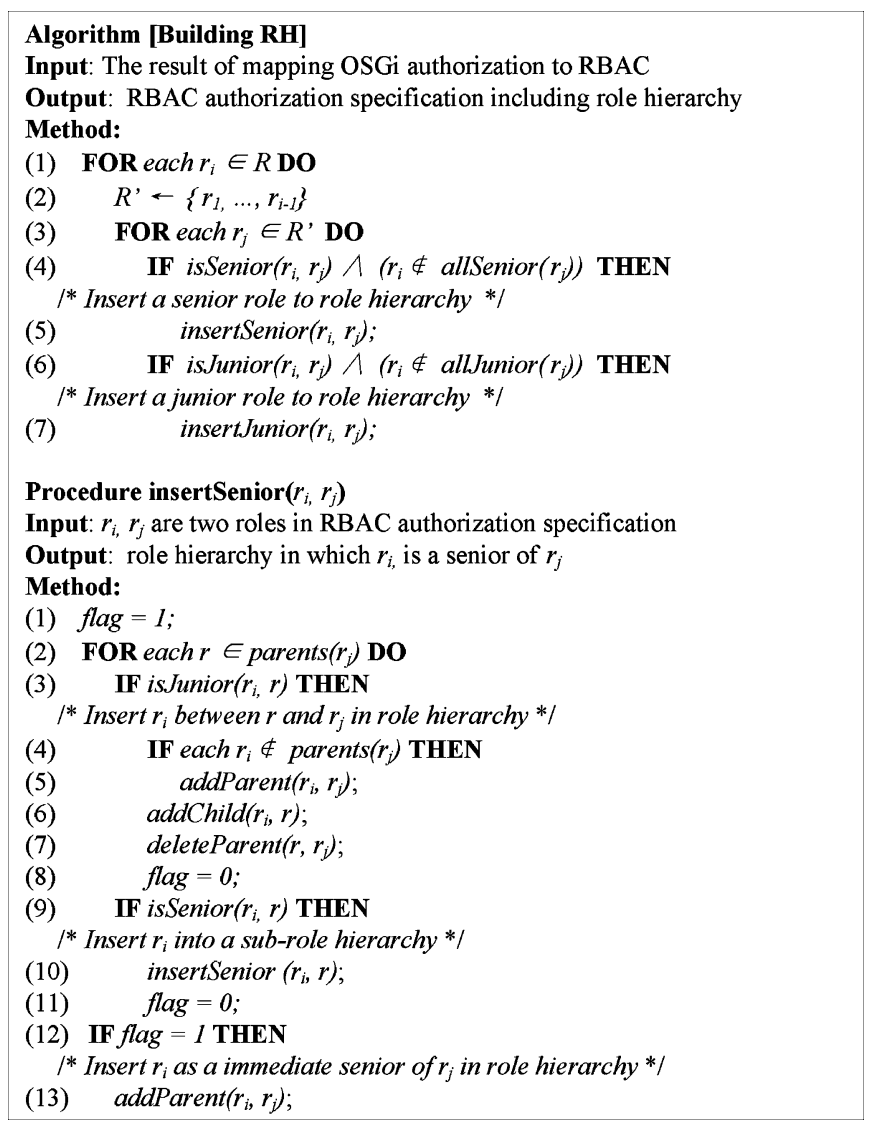

Fig. 5. Algorithm for building role hierarchy.

senior role, $r_{1}$ inherits all permissions of $r_{7}$. Commonly, if any of the following two conditions is true, $r_{i}$ is senior to $r_{j}$.

1) (ba_member $\left.\left(r_{j}\right) \neq \phi\right) \wedge\left(\right.$ ba_member $\left.\left(r_{i}\right) \neq \phi\right)$

$$
\begin{aligned}
& \wedge\left(\text { re_member }\left(r_{i}\right) \neq \phi\right) \\
& \wedge\left(\text { ba_member }\left(r_{j}\right)=\text { ba_member }\left(r_{i}\right)\right) \\
& \wedge\left(\text { re_members }\left(r_{j}\right) \subset \text { re_members }\left(r_{i}\right)\right) \\
\text { 2) } \quad & \left(\text { ba_member }\left(r_{j}\right)=\phi\right) \wedge\left(\text { re_member }\left(r_{i}\right) \neq \phi\right) \\
& \wedge\left(\text { re_member }\left(r_{j}\right) \neq \phi\right) \\
& \wedge\left(\text { re_members }\left(r_{j}\right) \subset \text { re_members }\left(r_{i}\right)\right) .
\end{aligned}
$$

In the first condition, $r_{i}$ has both a basic member and required members, and $r_{j}$ has at least a basic member. When $r_{i}$ and $r_{j}$ have same basic member, and the required member set of $r_{j}$ (including empty set) is a subset of the required member set of $r_{i}, r_{i}$ is senior to $r_{j}$. In another condition, if $r_{j}$ contains only a required member set, which is a subset of the required member set of $r_{i}, r_{j}$ is a junior role of $r_{i}$. With this methodology, two role hierarchies can be identified, as shown in Fig. 4(b).

We now introduce an algorithm for building role hierarchies described in Fig. 5. The algorithm builds role hierarchies through a series of continuous processes of adding a role to existing role hierarchies. The continuous processes are controlled by two loops that are called to compare each role $r_{i} \in R$ with every role $r_{j} \in\left\{r_{1}, \ldots, r_{i-1}\right\}$ in the existing role hierarchies. 
If $r_{i}$ is senior to $r_{j}$ and $r_{i}$ is not in role hierarchies, we can insert $r_{i}$ to role hierarchies as a senior of $r_{j}$ by calling insertSenior procedure. Another possible case is performing insert Junior procedure when $r_{i}$ is junior to $r_{j}$. The algorithm is illustrated in Fig. 5.

Supposing there exits a role hierarchy and $r_{j}$ is in the hierarchy, then we need to insert $r_{i}$ to the hierarchy as a senior role of $r_{j}$. There are three possible cases for the inserting process that are given as follows.

1) If $r_{i}$ is junior to a role $r \in$ parents $\left(r_{j}\right), r_{i}$ is inserted between $r$ and $r_{j}$.

2) If $r_{i}$ is senior to a role $r \in$ parents $\left(r_{j}\right), r_{i}$ should be inserted into a subrole hierarchy and a recursive process should be carried out.

3) If $r_{i}$ has no relations with all parent roles of $r_{j}, r_{i}$ is added into the role hierarchy as an immediate senior of $r_{j}$.

There are two kinds of assignment relations in RBAC: explicit assignment and implicit assignment. These assignments can result in redundant role memberships. To detect and eliminate the redundancies, the following step is also taken into account: if a user $u$ has been assigned to a superior role $r$ in role hierarchy, all user-to-role assignments between $u$ to junior roles of $r$ are examined.

3) Applying Well-Known Role-Based Constraints: RBAC is a policy-oriented approach, and allows to specify well-known security principles, such as separation of duty and least privilege. These security principles can be defined as constraints in RBAC. Also, we have proved that OSGi authorization requirements can be satisfied by RBAC in the previous discussion.

\section{B. Reconstructing OSGi Authorization From RBAC}

In the previous section, we show that OSGi authorization mechanism can be mapped to RBAC and it could be enhanced by important RBAC features. In this section, we discuss how we can reconstruct OSGi authorization mechanism from RBACbased authorization when we change PA and user-to-role assignment (UA). ${ }^{1}$ Note that in our approach, core RBAC components are constructed from OSGi authorization components and some OSGi authorization features are maintained in the constructed RBAC components bound to the private memberships. Thus, changes in RBAC components are constrained to maintain the consistency with OSGi authorization components. For this reason, we name RBAC, used in this paper, as an OSGi-compliant RBAC.

1) PA Change: In RBAC, an administrator can change the PA by adding or deleting an assignment relation. We first consider the ADD operation. Suppose an administrator attempts to assign a permission $p$ to a role $r$ in the OSGi-compliant RBAC. If the permission $p$ has not been assigned to any role, the ADD operation is allowed. To reflect this change in OSGi authorization, a new basic assignment ( $B$ in the AG-UG table) should be created between the action group ag corresponding to $p$ and the

\footnotetext{
${ }^{1}$ Some RBAC features, such as RBAC constraints, could not be recognized during reconstruction, since the original OSGi authorization mechanism cannot support these features. However, we attempt to apply such a crucial feature to our approach.
}

user group ug as the basic private member of $r$. In addition, required assignments ( $R$ in the AG-UG table) between the action group ag and all user groups as required private members of $r$ should be added as well.

If $p$ already has been assigned to other roles, we note that there are three potential cases from the previous construction processes.

1) The roles assigned to $p$ have both basic and required private members. And the required private member set of these roles should be the same. If $p$ is assigned to another role $r, r$ should also have both basic and required private members, and the required private members must be the same as the roles that $p$ has already been assigned to. Otherwise, the assignment operation is not allowed. In reflecting this new permission-role assignment, a basic assignment relation between the action group ag corresponding to $p$ and the user group as the basic private member of $r$ should be created in OSGi authorization.

2) All roles of $p$ have only a basic private member. If the administrator assigns $p$ to another role $r$, this role $r$ should also have only a basic private member. The corresponding basic assignment is created in OSGi authorization.

3) If $p$ has been assigned to a role and the role has only required private members, $p$ cannot be further assigned to other roles in RBAC.

Next, we consider the DELETE operation of an assignment relation between a permission $p$ and a role $r$ in RBAC. There are no constraints for revoking permission-role assignments in RBAC. When we reconstruct OSGi authorization, if $p$ has been assigned to roles other than $r$, we delete the basic assignment relation $(B)$ between the action group ag corresponding to $p$ and the user group ug as the basic member of $r$. For other case, if $p$ has been assigned only to $r$, then all assignment relations between the action group ag and the private members of $r$ in OSGi authorization are deleted. A detailed PA change algorithm is given in Fig. 6. This algorithm is used to reconstruct OSGi authorization when a PA is changed in RBAC. The complexity of PA change algorithm is $O\left(n_{\mathrm{UG}}\right)$, where $n_{\mathrm{UG}}$ represents the number of user groups in OSGi authorization.

2) UA Change: Similar to the PA change, UA change includes adding and/or deleting a user-to-role assignment under certain conditions in RBAC. When a user $u$ is assigned to a role $r$ in RBAC, the corresponding user in OSGi authorization should be assigned to all private members of the role in OSGi authorization. Thus, the assignment relation between $u$ and a private member $\operatorname{ug}_{i} \in$ members $(r)$ should be added if the user $u$ has not been assigned to $\mathrm{ug}_{i}$ in OSGi authorization.

Since a user-to-role assignment in RBAC is build based on the relations between the users and all private members of this role, deleting any of such relations may cause the revocation of the user-to-role assignment in RBAC. Thus, when an administrator wants to delete a user-to-role assignment in RBAC, we cannot just delete all relations between a particular user and all private members of this role directly in OSGi, and this might result in other UA changes to other roles. To reduce the complexity, our UA change algorithm requires the administrator to select the exact private members of the role that should be revoked. 


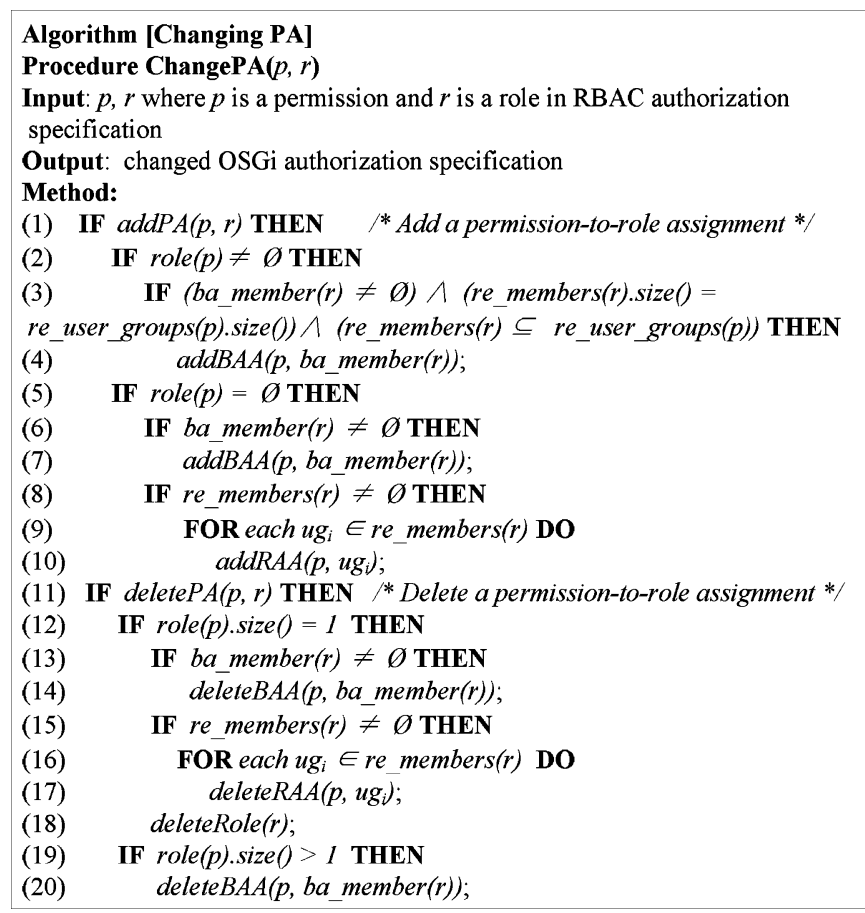

Fig. 6. Algorithm for handling PA change.

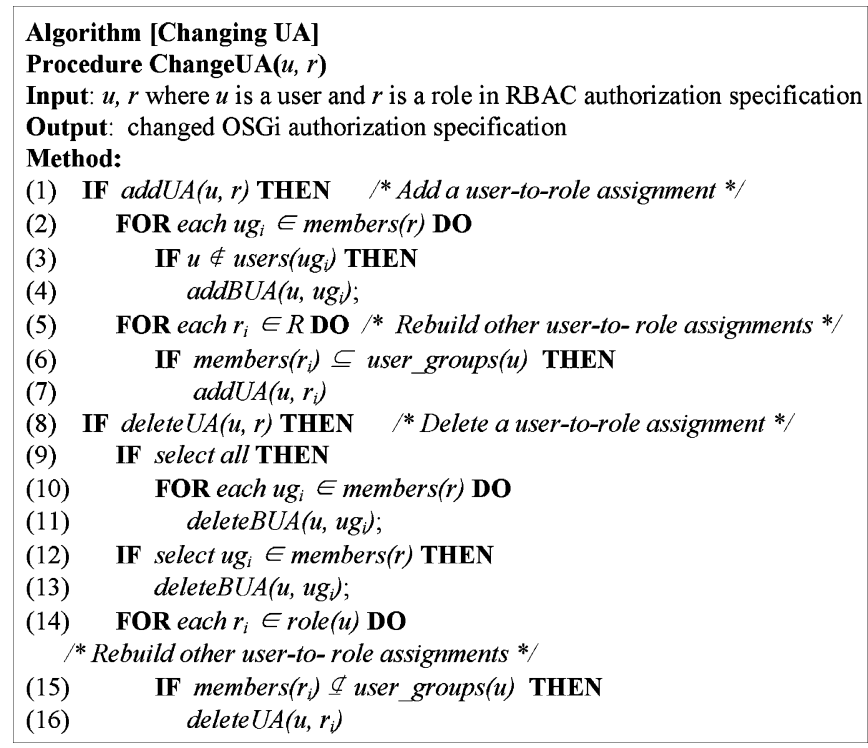

Fig. 7. Algorithm for handling UA change.

The revocations are directly reflected in the user-to-user group assignments in OSGi. Nevertheless, the changes of user-to-user group assignments in OSGi authorization may also affect other user-to-role assignments in RBAC. The user-to-role assignment relations in RBAC should be rebuild to be consistent with OSGi authorization changes. The rebuilding process can be performed through checking all roles that the user is assigned to. The UA change algorithm is shown in Fig. 7. Since the UA change algorithm involves two assignment processes, the complexity of the algorithm is $O\left(n_{\mathrm{UG}}+n_{R}\right)$, where $n_{\mathrm{UG}}$ and $n_{R}$ represent the number of user groups and roles in the authorization systems, respectively.

\section{IMPLEMENTATION AND PERFORMANCE EVALUATION}

To prove the feasibility of our approach, we design a prototype system, which is based on an open source OSGi framework implementation called Knopflerfish [2]. A security-enhanced OSGi authorization architecture is depicted in Fig. 8. In our implementation, a Web-based OSGi authorization management tool is designed to support our policy management framework. The tool is composed of two major GUIs: 1) OSGi authorization management GUI communicates with OSGi authorization engine to manage standard OSGi authorization policy ${ }^{2}$ and 2) RBAC authorization management GUI is used to manage RBAC authorization through the RBAC authorization engine. Transformation handler is responsible for mapping OSGi authorization to RBAC, building role hierarchies, and reconstructing OSGi authorization by performing corresponding algorithms. In addition, the policy enforcement is based on the pull mode, in which a policy enforcement point (PEP) collects the related information of subjects and queries a policy decision point (PDP) for policy decision. By integrating the Sun's XACML library [4] into OSGi service platform, the authorization service as the PDP module interprets XACML polices and makes access decisions. The User Admin service as the PEP module queries the authorization service and enforces the relevant operations.

Finally, we give a scenario to illustrate the usage of our prototype system. Supposing OSGi service platform is installed in a home network, which has been integrated with the implementation of our authorization framework. Bob is an administrator of the home network gateway. He defines an action group AccessWebCam, and two user groups, Residents, and Adults, as a basic member and a required member of the action group AccessWebCam, respectively. Then, when he uses our tool to map the OSGi authorization to RBAC, a role Residents_Adults is constructed and the permission corresponding to the action group AccessWebCam is assigned to the role. In addition, Bob defines several constraints for the role Residents_Adults. For example, a constraint can be established to specify "the user assigned to Residents_Adults role can perform the AccessWebCam action when he/she is in his/her office between 9 am and $5 \mathrm{pm}$."Now, Alice wants to access the webcam installed in the home remotely. She logs into the home service gateway and retrieves AccessWebCam bundle. If AccessWebCam bundle does not exist in the service gateway, she requests the service gateway to download AccessWebCam bundle from the bundle repository and install it in the gateway. However, when Alice tries to execute the AccessWebCam bundle, the system denies her access request and indicates that she needs permission for starting the bundle. There are two possible cases that cause such access denials. One case would be that $B o b$ has not assigned the user Alice to the two user groups, Residents and Adults, in OSGi authorization management or has not assigned Alice to Residents_Adults role in RBAC authorization management.

\footnotetext{
${ }^{2}$ The current OSGi authorization mechanism can coexist with the OSGicompliant RBAC authorization mechanism.
} 


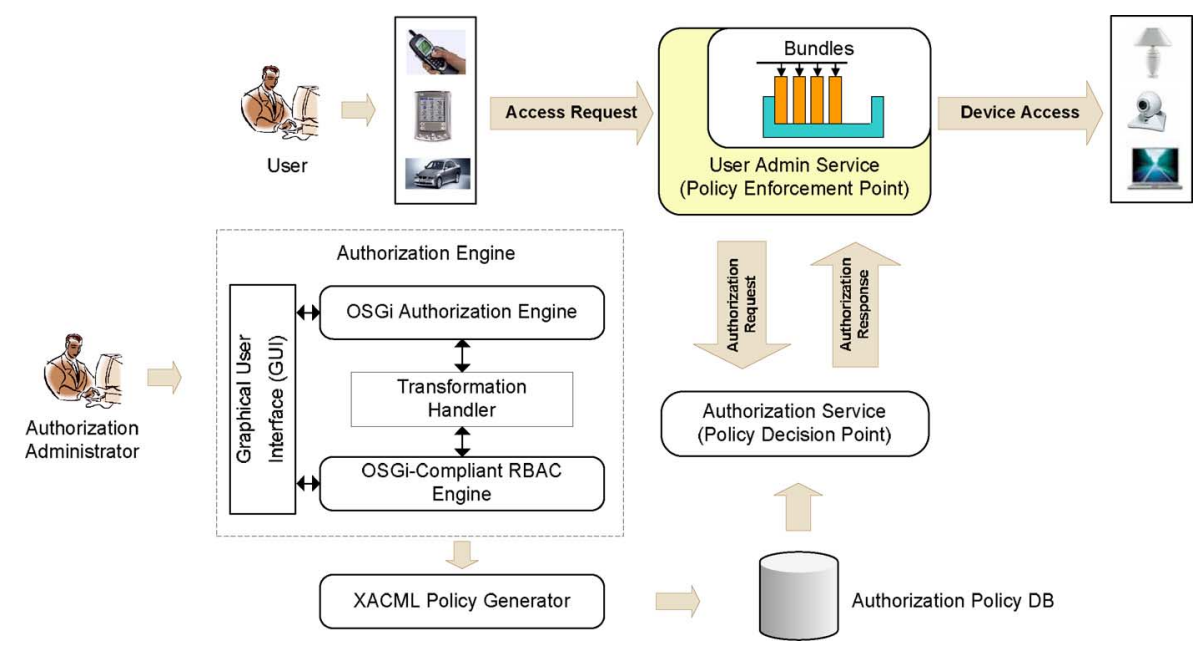

Fig. 8. Security-enhanced OSGi authorization architecture.

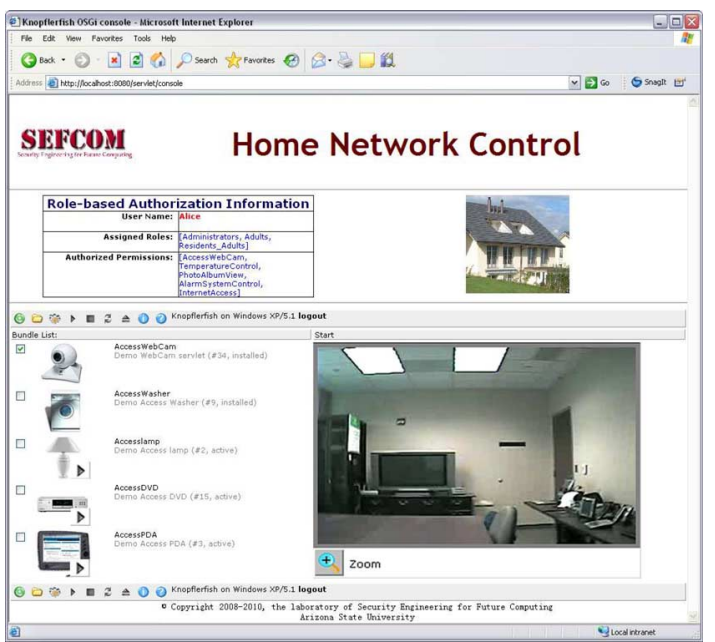

Fig. 9. Prototype system.

Another possible case is that the constraints are violated by Alice's current conditions. Hence, for the former case, Alice can ask $B o b$ to set up the corresponding assignment relations, while Alice can start AccessWebCam bundle when her situation satisfies the location and time constraints for the latter case. When all conditions are satisfied, Alice's remote access request to webcam is allowed, as shown in Fig. 9.

According to the rules for constructing OSGi-compliant RBAC in our solution, the number of generated roles and the number of PAs are determined by the number of basic assignment relationships (BAAs) between action groups and user groups. Obviously, BAA assignments in OSGi authorization policies affect the response time for our RBAC authorization system. Therefore, the number of BAA is chosen as the evaluation metrics for our experiments. By randomly triggering OSGi bundle access, we measure and compare the response time of three authorization mechanisms, as shown in Fig. 10. The experiments were performed on Intel Core 2 Duo CPU $3.00 \mathrm{GHz}$ with 3.25 GB RAM running on Windows XP and Apache Tomcat. Each result was measured in milliseconds and computed based

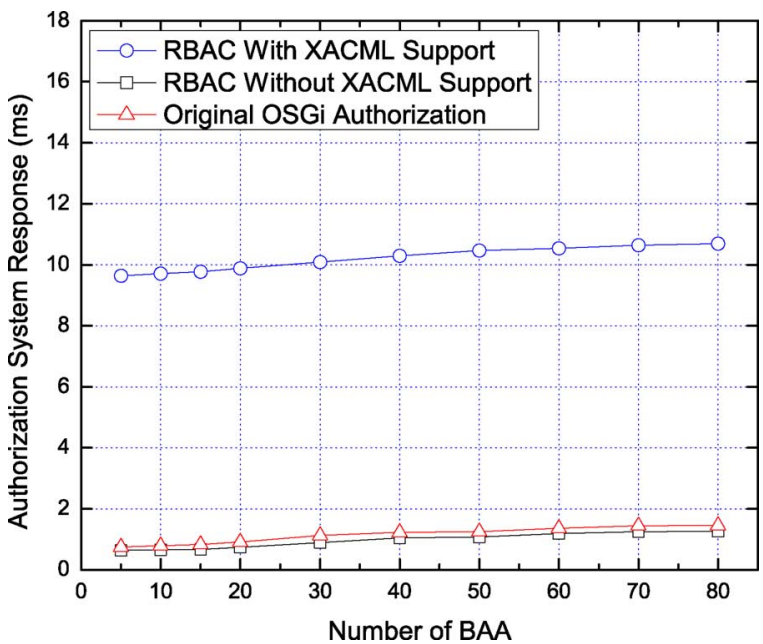

Fig. 10. Performance evaluation of the system implementation.

on the average over simulated runs. Compared to the original OSGi authorization mechanism, RBAC authorization without XACML shows better performance, since RBAC directly maps users to permissions through roles. In other words, our experiments prove that the proposed OSGi strategies are correctly designed. When RBAC policies are specified in XACML, it introduced about $9 \mathrm{~ms}$ overhead to RBAC authorization due to XACML request generation and policy evaluation. However, considering the potential benefits of XACML standard such as extensibility and interoperability, we believe this overhead is acceptable.

\section{CONCLUSION AND FutURE WORK}

In this paper, we have formulated current OSGi authorization mechanism and identified its inherent limitations. In addition, we have demonstrated how OSGi authorization mechanism could be mapped to RBAC and how OSGi authorization requirements can be fulfilled to leverage important RBAC features in the OSGi environment. Furthermore, we discussed possible enhancement of OSGi authorization with role-based constraints. 
A proof-of-concept prototype has been implemented to show the feasibility of our approach with possible consideration of interoperability and extensibility issues. Our future work would attempt to specify and verify complicated access control policies in OSGi environments, especially context-aware constraints including tools for policy specification and analysis. Also, we would study how our approach can be applied to universal plug and play (UPnP) [5] and digital living network alliance (DLNA) [1], which have similar security requirements as OSGi in home network environments.

\section{REFERENCES}

[1] Digital Living Network Alliance. (2003). [Online]. Available: http://www. dlna.org/home

[2] Knopflerfish. Knopflerfish Open Source OSGi. (2006). [Online]. Available: http://www.knopflerfish.org/index.html

[3] OSGi Alliance. OSGi Initiative. (1999). [Online]. Available: http://www. osgi.org.

[4] Sun Microsystems, Inc. Sun's XACML implementation. (2004). [Online]. Available: http://sunxacml.sourceforge.net/

[5] UPnP Forum. UPnP Standards for Device Security and Security Console. (2003). [Online]. Available: http://www.upnp.org/standardizeddcps/ security.asp

[6] G.-J. Ahn and R. S. Sandhu, "Role-based authorization constraints specification," ACM Trans. Inf. Syst. Security, vol. 3, no. 4, pp. 207-226, Nov. 2000.

[7] E. Bertino, L. Khan, R. Sandhu, and B. Thuraisingham, "Secure knowledge management: Confidentiality, trust, and privacy," IEEE Trans. Syst., Man, Cybern. A, Syst., Humans, vol. 36, no. 3, pp. 429-438, May 2006.

[8] E.-A. Cho, C.-J. Moon, D.-H. Park, and D.-K. Baik, "Access control policy management framework based on RBAC in OSGi service platform," in Proc. 6th IEEE Int. Conf. Comput. Inf. Technol. (CIT 2006), IEEE Computer Society, Washington, DC, 2006, pp. 161-166.

[9] E.-A. Cho, C.-J. Moon, D.-H. Park, and D.-K. Baik, "An effective policy management framework using RBAC model for service platform based on components," in Proc. 4th Int. Conf. Softw. Eng. Res., Manage. Appl. (SERA 2006), IEEE Computer Society, Washington, DC, 2006, pp. 281288.

[10] C. M. Ellison, "Home network security," Intel Technol. J., vol. 6, no. 4, pp. 37-48, 2002.

[11] T. Jaeger, "On the increasing importance of constraints," in Proc. 4th ACM Workshop Role-based Access Contr., 1999, pp. 33-42.

[12] S. Jajodia, P. Samarati, V. S. Subrahmanian, and E. Bertino, "A unified framework for enforcing multiple access control policies," in Proc. ACM SIGMOD Int. Conf. Manage. Data, 1997, pp. 474-485.

[13] C. Lee, D. Nordstedt, and S. Helal, "Enabling smart spaces with OSGi," IEEE Pervasive Comput., vol. 2, no. 3, pp. 94-98, Jul.-Sep. 2003.

[14] H.-Y. Lim, Y.-G. Kim, C.-J. Moon, and D.-K. Baik, "Bundle authentication and authorization using XML security in the OSGi service platform," in Proc. 4th Annu. ACIS Int. Conf. Comput. Inf. Sci. (ICIS 2005), IEEE Computer Society, Washington, DC, 2005, pp. 502-507.

[15] M. Lorch, D. B. Adams, D. Kafura, M. S. R. Koneni, A. Rathi, and S. Shah, "The prima system for privilege management, authorization and enforcement in grid environments," presented at the 4th Int. Workshop Grid Comput., Phoenix, AR, 2003.

[16] OASIS, XACML 2.0 Core: eXtensible Access Control Markup Language (XACML) Version 2.0. (2005). [Online]. Available: http://docs.oasisopen.org/xacml/2.0/access_control-xacml-2.0-core-spec-os.pdf

[17] OSGi, User Admin Service Specification Version 1.1. (2006). [Online]. Available: http://www.osgi.org

[18] R. Sandhu, "The ntree: A two dimension partial order for protection groups," ACM Trans. Comput. Syst., vol. 6, no. 2, pp. 197-222, May 1988.

[19] R. Sandhu, E. Coyne, H. Feinstein, and C. Youman, "Role-based access control model," IEEE Comput., vol. 2, no. 29, pp. 38-47, Feb. 1996.

[20] R. Sandhu and Q. Munawer, "How to do discretionary access control using roles," in Proc. 3rd ACM workshop Role-Based Access Contr., Aug. 1999, pp. 47-54.
[21] R. S. Sandhu, "Lattice-based access control models," IEEE Comput., vol. 26, no. 11, pp. 9-19, Nov. 1993

[22] R. S. Sandhu and P. Samarati, "Access control: Principles and practice," IEEE Commun. Mag., vol. 32, no. 9, pp. 40-48, Sep. 1994.

[23] C. Wu, C. Liao, and L. Fu, "Service-oriented smart-home architecture based on OSGi and mobile-agent technology," IEEE Trans. Syst., Man, Cybern. C, Appl. Rev., vol. 37, no. 2, pp. 193-205, Mar. 2007.

[24] H. Zhu and M. Zhou, "Roles in information systems: A survey," IEEE Trans. Syst., Man, Cybern. C, Appl. Rev., vol. 38, no. 3, pp. 377-396, May 2008

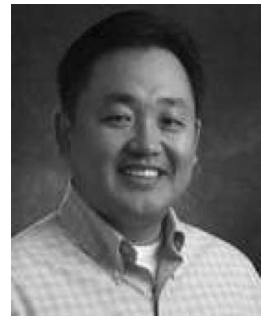

Gail-Joon Ahn (SM'07) received the Ph.D. degree in information technology from George Mason University, Fairfax, Virgina, 2000.

He was an Associate Professor in the College of Computing and Informatics, and the Founding Director of the Center for Digital Identity and Cyber Defense Research, and Laboratory of Information Integration, Security, and Privacy (LIISP), University of North Carolina at Charlotte, Charlotte. He is currently an Associate Professor in the Department of Computer Science and Engineering and the Director of Security Engineering for Future Computing (SEFCOM) Laboratory at Arizona State University (ASU), Tempe. His current research interests include information and systems security, vulnerability and risk management access control, and security architecture for distributed systems. His research has been supported by the U.S. National Science Foundation, National Security Agency (NSA), U.S. Department of Defense (DoD), U.S. Department of Energy (DoE), Bank of America, Hewlett Packard, Microsoft, and Robert Wood Johnson Foundation.

Dr. Ahn is a recipient of the U.S. Department of Energy CAREER Award and the Educator of the Year Award from the Federal Information Systems Security Educators Association (FISSEA).

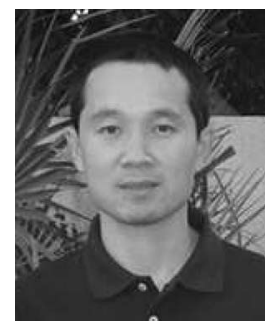

Hongxin $\mathrm{Hu}$ is currently working toward the $\mathrm{Ph} . \mathrm{D}$. degree at the Department of Computer Science and Engineering, Arizona State University, Tempe.

$\mathrm{He}$ is a member of the Security Engineering for Future Computing (SEFCOM) Laboratory, Arizona State University. His current research interest includes access control models and mechanisms, network and distributed system security, secure software engineering, and security in future home network.

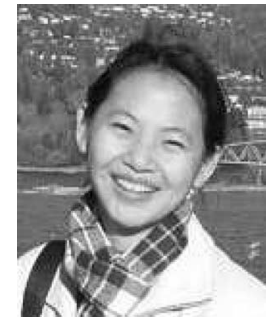

Jing Jin is currently working toward the Ph.D. degree at the College of Computing and Informatics, University of North Carolina at Charlotte, Charlotte.

She is a member of the Laboratory of Information Integration, Security, and Privacy (LIISP), University of North Carolina at Charlotte. Her current research interests include access control and trust management, identity and privacy management, network and distributed system security, and security in health informatics. 